\title{
ANALYTIC FUNCTIONS ON THE BIDISK AT BOUNDARY SINGULARITIES VIA HILBERT SPACE METHODS
}

\author{
RYAN TULLY-DOYLE
}

Abstract. We investigate the behavior of a generalized Hilbert space model of a function in the Schur class of the bidisk at singular boundary points that satisfy a growth condition. We examine the relationship between the boundary behavior of Schur functions and the geometry of corresponding generalized Hilbert space models. We describe a geometric condition on an associated operator that classifies the behavior of the directional derivative of the underlying Schur function at a carapoint.

Mathematics subject classification (2010): 32A30, 32S05, 30E20, 47A56, 47A57. behavior.

Keywords and phrases: Schur class, bidisk, Carathéodory condition, directional derivative, boundary

\section{REFERENCES}

[1] M. АвATE, The Julia-Wolff-Carathéodory theorem in polydisks, J. Anal. Math. 74 (1998), 275-306.

[2] J. AGLER, On the representation of certain holomorphic functions defined on a polydisc, Operator Theory: Advances and Applications, 48, (1990), 47-66.

[3] J. Agler And J. E. McCarthy, Pick Interpolation and Hilbert Function Spaces, American Mathematical Society, Providence, 2002.

[4] J. Agler, J. E. McCarthy, And N. J. Young, A Carathéodory theorem for the bidisk using Hilbert space methods, Math. Ann. 352, (2012), 581-624.

[5] J. Agler, R. Tully-Doyle, And N. J. Young, Boundary behavior of analytic functions of two variables via generalized models, Indag. Math. (N.S.) 23, (2012), 995-1027.

[6] J. A. BALL, I. GOHBERG, AND L. RODMAN, Interpolation of rational matrix functions, Birkhäuser, Basel, 1990.

[7] C. CARATHÉODORY, Über die Winkelderivierten von beschraänkten analytischen Funktionen, Sitzunber. Preuss. Akad. Wiss. (1929), 39-52.

[8] J. B. Conway, A Course in Functional Analysis, Springer, New York, second edition, 1997.

[9] F. JAFARI, Angular derivatives in polydisks, Indian J. Math. 35, (1993), 197-212.

[10] G. Julia, Extension nouvelle d'un lemme de Schwarz, Acta Math. 42, (1920), 349-355.

[11] G. KNESE, Regularity and integrability of rational functions, Proc. Lond. Math Soc.111, 1 (2015), 1261-1306.

[12] J. E. PASCOE, An inductive Julia-Carathéodory theorem for Pick functions in two variables, preprint.

[13] W. Rudin, Function Theory in the unit ball of $C^{n}$, Springer-Verlag, Berlin, 1980.

[14] K. WLODARCZYK Julia's lemma and Wolff's theorem for J*-algebras, Proc. Amer. Math. Soc. 99, (1987), 472-476.

[15] N. Young $\varphi$ need not be analytic at a C-point, private communication, (2012). 